\title{
Detection of Calcifications in Retinoblastoma Using Gradient- Echo MR Imaging Sequences: Comparative Study between In Vivo MR Imaging and Ex Vivo High-Resolution CT
}

F. Rodjan, P. de Graaf, P. van der Valk, T. Hadjistilianou, A. Cerase, P. Toti, M.C. de Jong, A.C. Moll, J.A. Castelijns, and P. Galluzzi, on behalf of the European Retinoblastoma Imaging Collaboration

\begin{abstract}
BACKGROUND AND PURPOSE: Intratumoral calcifications are very important in the diagnosis of retinoblastoma. Although CT is considered superior in detecting calcification, its ionizing radiation, especially in patients with hereditary retinoblastoma, should be avoided. The purpose of our study was to validate $\mathrm{T} 2 * \mathrm{WI}$ for the detection of calcification in retinoblastoma with ex vivo CT as the criterion standard.
\end{abstract}

MATERIALS AND METHODS: Twenty-two consecutive patients with retinoblastoma (mean age, 21 months; range, 1-71 months) with enucleation as primary treatment were imaged at $1.5 \mathrm{~T}$ by using a dedicated surface coil. Signal-intensity voids indicating calcification on $\mathrm{T} 2$ *WI were compared with ex vivo high-resolution CT, and correlation was scored by 2 independent observers as poor, good, or excellent. Other parameters included the shape and location of the signal-intensity voids. In 5 tumors, susceptibility-weighted images were evaluated.

RESULTS: All calcifications visible on high-resolution CT could be matched with signal-intensity voids on $\mathrm{T} 2{ }^{*} \mathrm{WI}$, and correlation was scored as excellent in 17 (77\%) and good in 5 (23\%) eyes. In total, 93\% (25/27) of the signal-intensity voids inside the tumor correlated with calcifications compared with none (0/8) of the signal-intensity voids outside the tumor. Areas of nodular signal-intensity voids correlated with calcifications in $92 \%$ (24/26), and linear signal-intensity voids correlated with hemorrhage in $67 \%(6 / 9)$ of cases. The correlation of signal-intensity voids on SWI was better in 4 of 5 tumors compared with T2*WI.

CONCLUSIONS: Signal-intensity voids on in vivo T2*WI correlate well with calcifications on ex vivo high-resolution CT in retinoblastoma. Gradient-echo sequences may be helpful in the differential diagnosis of retinoblastoma. The combination of funduscopy, sonography, and high-resolution MR imaging with gradient-echo sequences should become the standard diagnostic approach for retinoblastoma.

ABBREVIATIONS: $\mathrm{HRCT}=$ high-resolution $\mathrm{CT}$; SIV = signal-intensity void

$\mathbf{R}$ etinoblastoma is generally treated on the basis of funduscopic, sonography, and imaging findings without prior histopathologic confirmation of diagnosis. The prevalence of calcifications is approximately $85 \%{ }^{1}$ and is considered the key finding in differentiating retinoblastoma from simulating lesions (Coats disease, persistent hyperplastic primary vitreous, or toxocara en-

Received September 16, 2013; accepted after revision July 15, 2014. From the Departments of Radiology (F.R., P.d.G., M.C.d.J., J.A.C.), Pathology (P.v.d.V.), and Ophthalmology (A.C.M.), VU University Medical Center, Amsterdam, the Netherlands; and Departments of Ophthalmology (T.H.), Neuroimaging and Neurointerventional Unit (A.C., P.G.), and Pathology (P.T.), Azienda Ospedaliera e Universitaria, Santa Maria alle Scotte, Siena, Italy.

This work was supported by grants from the ODAS Foundation, Delft, the Netherlands; National Foundation for the Blind and Visually Impaired, Utrecht, the Netherlands; and Blindenhulp Foundation, Gravenhage, the Netherlands.

Please address correspondence to F. Rodjan, MD, Department of Radiology, Room 0 X 112, VU University Medical Center, Postbox 7057, 1007 MB Amsterdam, the Netherlands; e-mail: firazia.rodjan@slz.nl

http://dx.doi.org/10.3174/ajnr.A4163 dophthalmitis) in young children. ${ }^{2}$ Very rare lesions such as medulloepithelioma and retinocytoma may also have calcifications and hence occasionally cause difficulty with clinical and radiologic differentiation. ${ }^{3}$

Sonography is the most commonly used imaging technique for the evaluation of intraocular tumors. The combination of funduscopy and sonography allows the identification of calcifications in $91 \%-95 \%$ of all patients with newly diagnosed retinoblastoma. ${ }^{4}$ Sensitivity to small calcifications decreases, however, in the presence of massive retinal detachment, vitreous hemorrhage, and subretinal fluid, potentially hampering the confirmation of the diagnosis. In these complicated eyes, CT is generally the method of choice for studying intraocular calcifications, with reported sensitivities of $81 \%-96 \%{ }^{5}$ Its diagnostic performance in staging retinoblastoma disease extent is limited, however, and the theoretic increased risk of radiation-induced cataracts and fatal cancers in children who are exposed to ionizing radiation should 
be considered. ${ }^{6}$ Patients with hereditary retinoblastoma in particular are at an even higher risk of developing radiation-induced tumors compared with healthy children.

MR imaging is the noninvasive technique of choice for the evaluation of retinoblastoma. The combination of sonography and MR imaging is considered first-line diagnostic imaging in the evaluation of children with suspected retinoblastoma, surpassing CT. ${ }^{7}$ However, MR imaging does not allow reliable identification of tumoral calcifications on routinely used clinical sequences. The lack of spatial resolution with standard clinical sequences provides an additional challenge for the visualization of small punctate tumoral calcifications. Scarce data on high-resolution ocular MR imaging by using surface coils do, however, suggest that calcifications can be detected with reasonable diagnostic accuracy. ${ }^{8}$

Gradient-echo $\mathrm{T}^{*}$-weighted imaging sequences are sensitive to susceptibility differences among tissues that cause magnetic field inhomogeneity leading to signal loss, and T2*WI is used to depict blood products, deoxygenated venous blood in dilated vessels (venous congestion), and calcifications. ${ }^{9,10}$ Previous work by Galluzzi et al $^{11}$ showed that $\mathrm{T} 2{ }^{\star} \mathrm{WI}$ can be a feasible technique for detecting intraocular calcifications because calcified areas in retinoblastoma emerged as hypointense foci of signal-intensity voids (SIVs) within the soft-tissue mass. Most of the SIVs on T2*WI correlated with spots of intratumoral calcifications on CT. Whether the shape and spatial arrangement of the intraocular SIVs on MR imaging could be matched with calcifications on CT was not investigated.

The purpose of our study was to assess the performance of gradient-echo $\mathrm{T} 2^{\star} \mathrm{WI}$ in the visualization and morphologic evaluation of retinoblastoma calcifications and to compare $\mathrm{T} 2^{\star} \mathrm{WI}$ with ex vivo CT scans of the enucleated eyes as the criterion standard.

\section{MATERIALS AND METHODS Patient Population}

This study included patients from 2 European retinoblastoma referral centers and was performed in agreement with recommendations of both local ethics committees, with a waiver of informed consent. From October 2009 to September 2011, patients with retinoblastoma diagnosed with funduscopy and sonography under general anesthesia were included under the following conditions: 1) Adequate pretreatment $\mathrm{T} 2{ }^{\star} \mathrm{WI}$ was available, 2) enucleation of the eye was the primary treatment for retinoblastoma, and 3) high-resolution CT (HRCT) images of the enucleated eye were available. In patients with bilateral retinoblastoma, only the most affected eye was enucleated and included. Three patients were excluded because of inadequate $\mathrm{T} 2{ }^{\star} \mathrm{WI}$ quality. The final study population included 22 patients. Patient records were reviewed for age at retinoblastoma diagnosis.

\section{MR Imaging}

Pretreatment MR imaging examinations were performed with the patient under general anesthesia on $1.5 \mathrm{~T}$ systems (Avanto or Sonata; Siemens, Erlangen, Germany) by using a dedicated surface coil (loop or temporomandibular coils with a diameter of 4 and 7 $\mathrm{cm}$, respectively) focused on the affected or most affected eye. Imaging was performed according to published guidelines. ${ }^{7}$ In all patients, MR images included transverse and sagittal spin-echo T1WI (TR/TE, 300-420/13-15 ms; section thickness, $2 \mathrm{~mm}$ ), transverse spin-echo T2WI (TR/TE, 1200-2470/120-166 ms; section thickness, $2 \mathrm{~mm})$, and transverse $2 \mathrm{D} \mathrm{T} 2^{\star} \mathrm{WI}(\mathrm{TR} / \mathrm{TE}$, 300-650/15-25 ms; section thickness, $2 \mathrm{~mm}$ ). Additionally, 5 patients underwent susceptibility-weighted imaging (TR/TE, 46/38 ms; matrix, $192 \times 162$; voxel size, $0.4 \times 0.6 \times 1 \mathrm{~mm}^{3}$; no gap; 35 sections; averages, 2 ; flip angle, $15^{\circ}$; acquisition time, $7.34 \mathrm{~min}-$ utes). SWI datasets included phase images and minimum-intensity-projection images.

\section{Ex Vivo HRCT}

HRCT was performed immediately after enucleation by using either a BrightSpeed (GE Healthcare, Milwaukee, Wisconsin) or Somatom Sensation 64 (Siemens) system. Eyes were carefully positioned in a small cardboard tray supported with gauze in the same orientation as the transverse plane of the MR images. Axial images were acquired with a section thickness of $0.6 \mathrm{~mm}$, collimation of $0.5 \mathrm{~mm}$, pitch of $0.8,120 \mathrm{kV}, 250 \mathrm{mAs}$, FOV of $16 \mathrm{~cm}$, and a $512 \times 512$ matrix. From the raw data, individual datasets were reformatted into images of $0.6-\mathrm{mm}$ section thickness in all 22 eyes. Raw data remained available for additional multiplanar reconstructions, which were made afterward by 1 observer (F.R.) to provide accurate information of the obtained data in the axial plane. Finally, 2 datasets of CT images were reconstructed and were available for all patients; one with section thickness of $2 \mathrm{~mm}$ (the same orientation and section-thickness as the $\mathrm{T} 2{ }^{\star} \mathrm{WI}$ ) and one with very thin sections $(0.6 \mathrm{~mm})$.

\section{Image Analysis}

Retrospectively, 2 independent observers (P.G. and P.d.G, with 15 and 11 years of experience in ocular MR imaging, respectively) reviewed all $\mathrm{T} 2{ }^{\star} \mathrm{WI}$ and $\mathrm{HRCT}$ images. Afterward, differences in scoring were resolved in consensus. Both observers were blinded to the results of clinical and histopathologic findings. Ex vivo HRCT was considered the criterion standard for detecting calcifications. The presence or absence of calcifications on HRCT was assessed. Calcifications were defined as hyperattenuated foci within the tumor on HRCT images. MR images were independently reviewed by the same observers. After analyzing the MR images, the observers confirmed detection of calcifications in correlation with HRCT images by using anatomic landmarks to adjust for section thicknesses, angulation, and obliquity. Criteria used to define calcifications on MR imaging were as follows: Calcifications were required to have the same morphologic pattern as that on the HRCT (same shape and same spatial arrangement) and were considered localized foci of marked hypointense SIV on $\mathrm{T} 2{ }^{\star} \mathrm{WI}$. Correspondence between intraocular hyperattenuated areas on CT and intraocular SIVs observed on MR imaging was scored as excellent, good, or poor. Excellent correspondence was scored if all calcifications on HRCT perfectly matched SIVs on $\mathrm{T} 2{ }^{\star} \mathrm{WI}$. Good correspondence was scored if there was an evident correlation between HRCT and MR imaging in some parts of the tumor but additional SIVs on MR imaging were present without an evident explanation on the basis of other MR images or hyperattenuated structures on CT. If no correlation could be observed at all, the correspondence was scored as poor. The location of SIVs 
Patient findings and correlation of CT with MRI in calcium detection

\begin{tabular}{ccclcc}
\hline $\begin{array}{c}\text { Patient } \\
\text { (Lat) }\end{array}$ & $\begin{array}{c}\text { Age } \\
\text { (mo) }\end{array}$ & $\begin{array}{c}\text { Int MRI-En } \\
\text { (day) }\end{array}$ & $\begin{array}{c}\text { Corr } \\
\text { T2*WI-CT }\end{array}$ & $\begin{array}{c}\text { SWI } \\
\text { T2*WI-SWI }\end{array}$ \\
\hline $1(U)$ & 1 & 1 & Moderate & No & NA \\
$2(U)$ & 4 & 8 & Moderate & No & NA \\
$3(U)$ & 46 & 6 & Moderate & No & NA \\
$4(U)$ & 3 & 0 & Moderate & No & NA \\
$5(U)$ & 26 & 6 & Moderate & No & NA \\
$6(U)$ & 73 & 1 & Good & No & NA \\
$7(B)$ & 8 & 4 & Good & No & NA \\
$8(U)$ & 11 & 5 & Good & No & NA \\
$9(U)$ & 8 & 1 & Good & No & NA \\
$10(U)$ & 16 & 1 & Good & No & NA \\
$11(U)$ & 3 & 8 & Good & No & NA \\
$12(B)$ & 12 & 8 & Good & No & NA \\
$13(B)$ & 35 & 8 & Good & No & NA \\
$14(U)$ & 29 & 1 & Good & No & NA \\
$15(B)$ & 29 & 8 & Good & No & NA \\
$16(B)$ & 13 & 6 & Good & No & NA \\
$17(U)$ & 45 & 8 & Good & No & NA \\
$18(U)$ & 5 & 8 & Good & Yes & Equal \\
$19(U)$ & 9 & 7 & Good & Yes & Better \\
$20(U)$ & 38 & 1 & Good & Yes & Better \\
$21(U)$ & 5 & 8 & Good & Yes & Better \\
$22(U)$ & 37 & 5 & Good & Yes & Better \\
\hline
\end{tabular}

Note:-Lat indicates tumor laterality; Int MRI-En, interval MRI and enucleation; Corr, correlation; $\mathrm{U}$, unilateral; B, bilateral; NA, not applicable.

${ }^{a}$ Median age, 12.5 mo; mean age, 20.72 mo.

in the tumor was categorized as central, peripheral, or a combination of both. The shape of SIVs was categorized as nodular or linear. SIVs on $\mathrm{T} 2{ }^{\star} \mathrm{WI}$ secondary to intravitreal or subretinal hemorrhage were considered if fluid-fluid levels (in correspondence with T2WI) or a smooth hypointense outlining of the tumor or retina was present in the affected eye.

Eyes with good or poor correlation based on additional SIVs without corresponding calcification on HRCT or other explanations on conventional MR images were selected for additional correlation with histopathology. In 5 tumors, SIVs in SWI were evaluated and compared with the $\mathrm{T} 2{ }^{\star} \mathrm{WI}$ and were scored as worse, equal, or better correlation with calcifications on HRCT. Furthermore, the feasibility of using phase images for the detection of calcifications in retinoblastoma was studied. Calcium undergoes a positive phase shift (paramagnetic susceptibility) and is displayed as a high signal-intensity area on the phase image, whereas a negative phase shift (diamagnetic susceptibility) occurs for veins, iron, and hemorrhage, making them appear uniformly dark.

\section{Histopathologic Examination}

Eyes were fixed in saline-buffered formalin, sampled, embedded in paraffin, and sectioned in the same orientation as the axial plane of the MR images (thickness, $4 \mu \mathrm{m}$ ) and stained with hematoxylin-eosin. Histopathologic sections were only evaluated in tumors with additional SIVs on T2*WI, without evident cause on other MR images or CT, by 2 pathologists (P.v.d.V. and P.T).

\section{RESULTS}

All 22 patients (22 eyes) in this study had histopathologically proved retinoblastoma (mean age, 21 months; range, 1-73

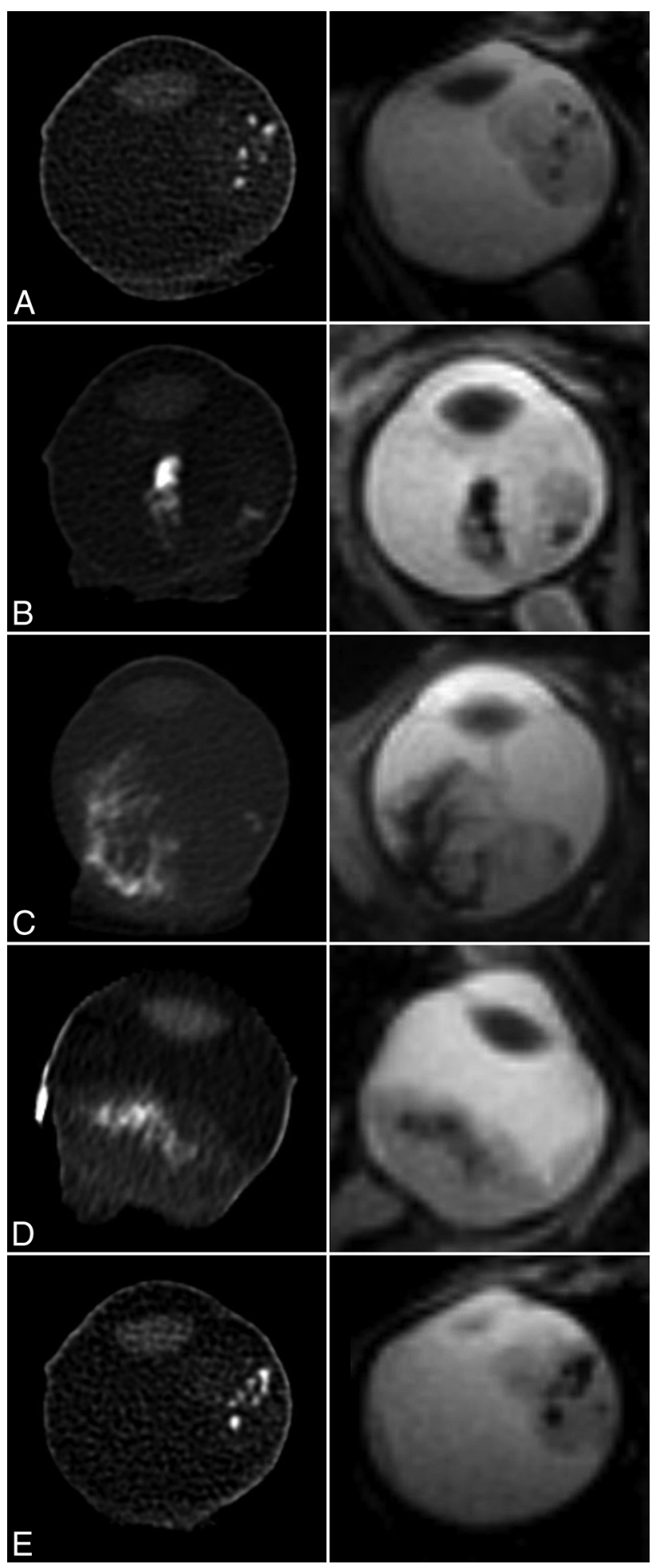

FIG 1. Excellent-matching hyperattenuated calcifications on ex vivo high-resolution CT (left column) with signal-intensity void spots on gradient-echo T2*-weighted MR images (right column) in patients 12 $(A), 16(B), 18(C), 19(D)$, and $14(E)$.

months). The mean time interval between diagnosis and MR imaging was 5 days (range, $0-8$ days). The mean time interval between MR imaging and CT was 5 days (range, $0-8$ days). Patient characteristics are summarized in the Table.

Areas of calcification were present on HRCT in the tumors of

AJNR Am J Neuroradiol 36:355-60 Feb 2015 www.ajnr.org 

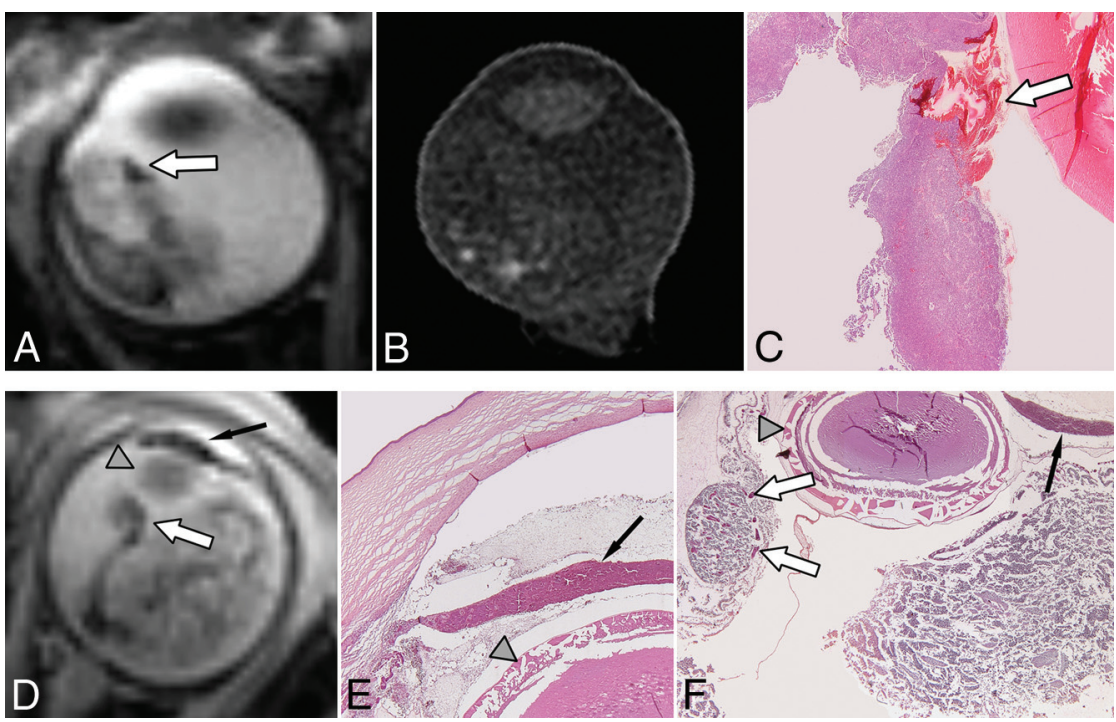

FIG 2. Examples of additional signal-intensity voids on $T 2^{*}$-weighted MR images without correspondence with ex vivo high-resolution CT. Patient 13 shows a hypointense nodular structure $(A)$ in the anterior part of the eye (arrow) on $\mathrm{T}^{*}$-weighted imaging without corresponding hyperattenuation on ex vivo high-resolution CT (B). Histopathology demonstrates a hemorrhage (arrow) precisely matching this additional SIV (C). Adjacent to this hemorrhage multiple lineararranged spots match hyperattenuated spots on CT. In patient $11(D)$, a linear band of SIV on T2*WI is shown outside the tumor along the detached retina (white arrow) and in the iris (black arrow). The gray arrowhead indicates the lens, which is dislocated. Histology ( $E$ and $F$ ) shows necrotic tumor with dilated vessels (venous congestion) (white arrow) and hemorrhagic necrosis of the iris combined with venous congestion (black arrow). The anterior chamber is infiltrated by neoplastic cells and cellular debris. (H\&E staining, $\times 20$ magnification.)
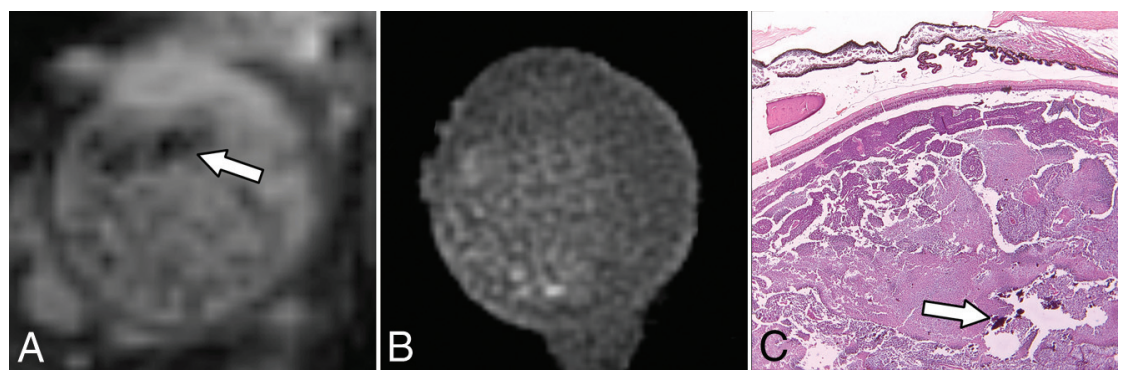

FIG 3. Extra signal-intensity void spots in the anterior part of the eye on gradient-echo $T 2^{*}$ weighted images (arrow, A) were observed in patient 21, with excellent correspondence with HRCT $(B)$. However, a band of additional signal-intensity void spots was present in the anterior part of the tumor on the gradient-echo T2*-weighted MR image (arrow, A) without correspondence on ex vivo high-resolution CT. Histopathologic correlation (C) shows multiple foci of calcifications in the anterior part of the tumor (arrow). (H\&E staining, $\times 20$ magnification.)

all 22 eyes. Furthermore, all eyes showed foci of SIVs on T2*WI. Thirty-five areas of SIVs were depicted, 27 areas of SIVs inside the tumor and 8 areas outside the tumor. Shape was classified as nodular $(n=26)$ and linear $(n=9)$. Nodular SIVs were predominantly located within the tumor (25 of 26 SIVs; $96 \%$ ), and 19 (76\%) were in the tumor center, whereas linear SIVs were predominantly located outside the tumor (7 of 9 SIVs; $78 \%$ ). Of the 2 linear SIVs within the tumor, one was located at the periphery of the tumor, while the other was located centrally within the tumor.

Correlation between calcifications on $\mathrm{CT}$ and SIVs on $\mathrm{T} 2{ }^{*} \mathrm{WI}$ was scored as excellent in $17(77 \%)$ and good in $5(23 \%)$ eyes. Poor correlations were not observed. Of 27 areas of SIVs located inside the tumor, 25 areas (93\%) correlated with calcifications; 1 , with intratumoral hemorrhage; and 1 , with a dilated venous vessel (venous congestion). Of the 8 areas of SIVs located outside the tumor, 6 (75\%) correlated with hemorrhage (5 subretinal and 1 intratumoral), and 2, with susceptibility artifacts secondary to air-tissue interface. Areas of nodular SIVs correlated with calcifications in $92 \%$ (24/26), and linear SIVs correlated with hemorrhage in $67 \%(6 / 9)$.

The 17 excellent-corresponding eyes showed a perfect match (same shape and same spatial arrangement) between hyperattenuated structures on $\mathrm{CT}$ and areas of SIVs on T2*WI (Fig 1). Additional SIVs in excellent-corresponding tumors were mostly located outside the tumor, with a linear aspect corresponding with artifacts or hemorrhage (Fig 2). In 3 excellent-corresponding tumors, additional SIVs were observed on $\mathrm{T} 2{ }^{*} \mathrm{WI}$ and did not have any corresponding hyperattenuation on HRCT. However on histopathology, these SIVs did correlate with spots of calcification (Fig 3).

In 5 good-corresponding eyes, the hypointense areas on MR imaging could only be correlated in part with hyperattenuated areas on CT. Additional SIVs were all located outside the tumor and correlated with hemorrhage and venous congestion.

Discrepancies between observers occurred in 2 excellent-corresponding cases. In one case, a linear hypointense structure that indicated hemorrhage caused confusion. In the other case, more SIVs were observed on MR imaging compared with the poor resolution of CT. The spots of calcification on CT, however, matched those on $\mathrm{T} 2{ }^{*} \mathrm{WI}$ well.

In 5 patients, SWI datasets were available and all eyes were excellent-corresponding. In 4 eyes, the correlation between SIVs on SWI and the calcifications on HRCT was better compared with that on $\mathrm{T} 2^{*} \mathrm{WI}$ (Fig 4 ), and in 1 eye, the correlation showed no difference between the 2 gradient-echo techniques. In 2 patients, the quality of the phase images was diminished due to image distortions. Phase images showed the hyperintense signal intensity of calcification in the other 3 eyes, which was confirmed on HRCT. None of these eyes showed intraocular or intratumoral hemorrhage clinically or on histopathology.

\section{DISCUSSION}

$\mathrm{T} 2^{\star} \mathrm{WI}$ allows accurate identification of calcified areas in retinoblastoma. In our study, all calcifications seen on ex vivo HRCT could be matched with SIVs on $\mathrm{T} 2^{*} \mathrm{WI}$, and this technique even depicted small SIVs better than HRCT. SIVs located in the tumor 


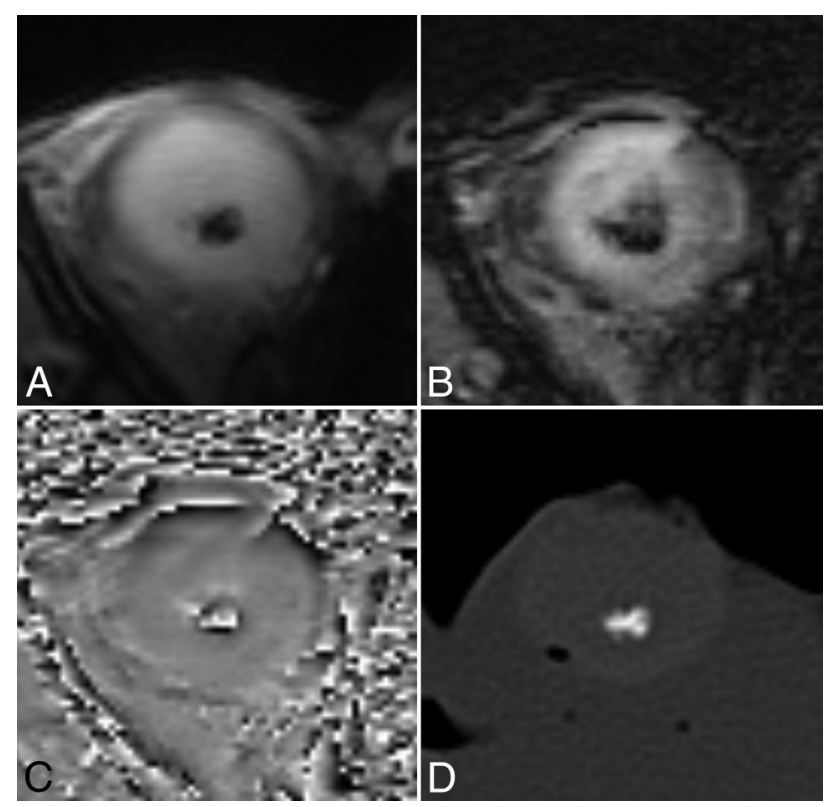

FIG 4. The value of phase imaging in identifying calcification in retinoblastoma (patient 22). Signal-intensity void spots can be seen on the $T 2^{*}$-weighted $(A)$ and SWI minimum intensity projection $(B)$. A phase image $(C)$ shows high signal intensity centrally identifying calcification, confirmed on the ex vivo high-resolution CT image $(D)$.

center are more likely to be calcification, whereas peripheral SIVs and other intraocular SIVs can also indicate hemorrhage, slowflowing blood in venous congestion, or artifacts.

Detection of calcifications is critical for the differential diagnosis of retinoblastoma. After funduscopy, the first-line diagnostic tool is always ocular sonography. Experienced ophthalmologists find calcifications in most patients. When calcifications are not detected, cross-sectional imaging studies can be used as a problem-solving technique. ${ }^{12,13} \mathrm{MR}$ imaging is the technique of choice for the evaluation of intraocular pathology, especially in children presenting with leukocoria.

The guidelines for imaging retinoblastoma by de Graaf et $\mathrm{al}^{7}$ serve as a checklist for minimal requirements for pretreatment diagnostic evaluation of retinoblastoma or mimicking lesions. They concluded that with sonography, high-resolution MR imaging is the most important technique for retinoblastoma diagnosis and the evaluation of associated intracranial abnormalities. CT was highly discouraged in children with retinoblastoma because of ionizing radiation and no added diagnostic value.

Historically, MR imaging has been considered an inferior technique for the detection of calcifications compared with CT. However, successful depiction of small foci of calcifications by $\mathrm{T} 2{ }^{\star} \mathrm{WI}$ has already been reported in other studies. ${ }^{14,15}$ Galluzzi et $\mathrm{al}^{11}$ showed that $\mathrm{T} 2{ }^{\star} \mathrm{WI}$ is a feasible technique for detecting calcifications in retinoblastoma. In their study, all SIVs matched calcifications. SIVs on T2*WI could, however, also represent pathology other than calcifications. In our study, we further characterized SIVs as calcification, hemorrhage, or artifacts by discussing different accompanying patterns.

We were able to confirm the capability of $\mathrm{T} 2{ }^{\star} \mathrm{WI}$ for calcification detection. In some tumors, $\mathrm{T} 2^{\star} \mathrm{WI}$ was even more sensitive in depicting small intraocular calcifications in vivo compared with
HRCT ex vivo. The HRCT protocol in this study is considered more sensitive for the detection of small punctate calcifications than commonly used clinical protocols for pediatric orbital CT.

Additional hypointense areas on MR imaging can, however, cause confusion. Intratumoral hemorrhage is the most important cause of false-positive SIVs. ${ }^{16}$ Hemorrhage is more likely to appear in large necrotic tumors and can cause extensive intraocular complications such as massive subretinal or intravitreal hemorrhage with a subsequent increase in intraocular pressure. Smooth and linear SIVs in retinoblastoma on $\mathrm{T} 2{ }^{*} \mathrm{WI}$ are indicative of intratumoral hemorrhage and are predominantly located in the tumor periphery, whereas calcifications more frequently present as hypointense SIVs in the tumor center. Massive subretinal or intravitreal hemorrhage can easily be diagnosed on conventional T2WI with fluid-fluid (sedimentation) levels. We considered smooth and linear SIVs on T2*WI indicative of intraocular hemorrhage. They are predominantly on the surface of the tumors or aligning with the usually detached retina. Close correlation with findings during funduscopy and sonography might further help in the interpretation of SIVs on the tumor surface.

Advanced disease with massive tumor necrosis can also present with secondary neovascular glaucoma, uveitis, and/or aseptic orbital cellulitis. These conditions are associated with venous congestion (ie, extremely dilated intraocular venous structures). Susceptibility effects in venous blood are caused by the presence of deoxyhemoglobin and become more pronounced in venous congestion with increased intravascular space and slow-flowing venous blood (Fig 4). ${ }^{17}$ Susceptibility artifacts due to air beneath the eyelid or within the paranasal sinuses can also cause SIVs on $\mathrm{T} 2{ }^{\star} \mathrm{WI}$. These artifacts can be distinguished from calcifications by their linear aspect and location. Susceptibility artifacts caused by air are usually located in or near the anterior eye segment.

SWI was available for review in a small subset of patients, which allowed us to explore, for the first time, the potential value of this sequence in retinoblastoma. This technique was more sensitive than $\mathrm{T} 2^{\star} \mathrm{WI}$ in detecting calcifications or microcalcifications and differentiating them from intratumoral hemorrhage or microhemorrhage, necrosis, and artifacts in oligodendroglioma, vestibular schwannoma, and diffuse infiltrating pontine glioma. ${ }^{10,18,19}$ In contrast to T2*WI, SWI is based on a long TE, high-resolution, flow-compensated $3 \mathrm{D}$ gradient-echo imaging technique with filtered phase information in each voxel. The combination of magnitude and phase data creates an enhanced contrast magnitude image that is particularly sensitive to hemorrhage, calcium, iron storage, and slow-flowing venous blood, therefore allowing a significant improvement in sensitivity and specificity compared with T2*WI. ${ }^{18,20}$ Preliminary results in our study showed that correlations between SWI and HRCT in retinoblastoma are equal to or even better compared with $\mathrm{T} 2{ }^{\star} \mathrm{WI}$. Therefore, further studies with more patients are required to determine the diagnostic accuracy of SWI in the detection of microcalcifications in retinoblastoma.

Our study has some limitations. First, we did not perform histopathologic correlation to confirm the presence of calcifications in all eyes but only of SIVs for which a corresponding hyperattenuation on HRCT was lacking. However, the accuracy of CT in depicting calcifications in soft tissues is well-accepted, and 
this technique was used as a reference in several studies in the past. ${ }^{5,11,21}$ Second, the lesions studied were all retinoblastomas, without a number of simulating lesions for comparison. It is thus impossible to determine whether the absence or theoretic absence of SIVs on T2*WI or SWI in simulating diseases can be a reliable parameter to narrow the differential diagnosis. Third, all included patients required enucleation, which resulted in selection bias because small tumors are usually treated with conservative (eyesparing) treatment options. The amount of calcifications might be higher in larger tumors, which makes detection of calcification by CT and MR imaging easier. A potential reduction in sensitivity might become apparent for both techniques by adding smaller tumors.

We recommend further research on this topic, in which retinoblastoma and simulating lesions are studied with $\mathrm{T} 2{ }^{\star} \mathrm{WI}$ or SWI to evaluate the presence of SIVs in these diseases. Ideally, these studies should include close correlation with histopathology to explore possible false-positive findings in simulating lesions. The small sample size of SWI studies in retinoblastoma is another limitation in our study. However, these initial positive findings warrant further research, especially because SWI, by including information provided by the phase images, has the potential to be a sensitive and specific technique for detecting or excluding calcifications in retinoblastoma.

\section{CONCLUSIONS}

Our study shows an excellent correlation between SIVs as detected on in vivo T2*WI or SWI sequences and calcifications on ex vivo HRCT in retinoblastoma. The combination of funduscopy, sonography, and high-resolution MR imaging with gradient-echo sequences should become the standard diagnostic approach for retinoblastoma and removes potentially harmful ionizing radiation from the study protocol.

Disclosures: Paolo Galluzzi—RELATED: Support for Travel to Meetings for the Study or Other Purposes: ODAS Foundation (from Italy to the Netherlands, for the European Retinoblastoma Imaging Collaboration meeting in Amsterdam).

\section{REFERENCES}

1. Levy J, Frenkel S, Baras M, et al. Calcification in retinoblastoma: histopathologic findings and statistical analysis of $\mathbf{3 0 2}$ cases. $\mathrm{Br} \mathrm{J}$ Ophthalmol 2011;95:1145-50

2. Chung EM, Specht CS, Schroeder JW. From the archives of the AFIP: pediatric orbit tumors and tumorlike lesions-neuroepithelial lesions of the ocular globe and optic nerve. Radiographics 2007;27: $1159-86$

3. Saunders T, Margo CE. Intraocular medulloepithelioma. Arch Pathol Lab Med 2012;136:212-16

4. Roth DB, Scott IU, Murray TG, et al. Echography of retinoblastoma: histopathologic correlation and serial evaluation after globe-con- serving radiotherapy or chemotherapy. J Pediatr Ophthalmol Strabismus 2001;38:136-43

5. Beets-Tan RG, Hendriks MJ, Ramos LM, et al. Retinoblastoma: CT and MRI. Neuroradiology 1994;36:59-62

6. Brenner D, Elliston C, Hall E, et al. Estimated risks of radiationinduced fatal cancer from pediatric CT. $A J R A m J$ Roentgenol 2001;176:289-96

7. de Graaf P, Göricke S, Rodjan F, et al. Guidelines for imaging retinoblastoma: imaging principles and MRI standardization. $P e-$ diatr Radiol 2012;42:2-14

8. Lemke AJ, Kazi I, Mergner U, et al. Retinoblastoma: MR appearance using a surface coil in comparison with histopathological results. Eur Radiol 2007;17:49-60

9. Chavhan GB, Babyn PS, Thomas B, et al. Principles, techniques, and applications of $\mathrm{T} 2^{\star}$-based MR imaging and its special applications. Radiographics 2009;29:1433-49

10. Zulfiqar M, Dumrongpisutikul N, Intrapiromkul J, et al. Detection of intratumoral calcification in oligodendrogliomas by susceptibilityweighted MR imaging. AJNR Am J Neuroradiol 2012;33:858-64

11. Galluzzi P, Hadjistilianou T, Cerase A, et al. Is CT still useful in the study protocol of retinoblastoma? AJNR Am J Neuroradiol 2009; 30:1760-65

12. de Graaf P, van der Valk P, Moll AC, et al. Retinal dysplasia mimicking intraocular tumor: MR imaging findings with histopathologic correlation. AJNR Am J Neuroradiol 2007;28:1731-33

13. Mafee MF, Goldberg MF, Cohen SB, et al. Magnetic resonance imaging versus computed tomography of leukocoric eyes and use of in vitro proton magnetic resonance spectroscopy of retinoblastoma. Ophthalmology 1989;96:965-75

14. Fatemi-Ardekani A, Boylan C, Noseworthy MD. Identification of breast calcification using magnetic resonance imaging. Med Phys 2009;36:5429-36

15. Tsushima $Y$, Endo K. Hypointensities in the brain on $\mathbf{T} 2^{*}$-weighted gradient-echo magnetic resonance imaging. Curr Probl Diagn Radiol 2006;35:140-50

16. Atlas SW, Grossman RI, Hackney DB, et al. Calcified intracranial lesions: detection with gradient-echo-acquisition rapid MR imaging. AJR Am J Roentgenol 1988;150:1383-89

17. Kvistad KA, Rydland J, Vainio J, et al. Breast lesions: evaluation with dynamic contrast-enhanced T1-weighted MR imaging and with T2*-weighted first-pass perfusion MR imaging. Radiology 2000;216: $545-53$

18. Löbel U, Sedlacik J, Sabin ND, et al. Three-dimensional susceptibility-weighted imaging and two-dimensional $\mathrm{T} 2{ }^{\star}$-weighted gradient-echo imaging of intratumoral hemorrhages in pediatric diffuse intrinsic pontine glioma. Neuroradiology 2010;52:1167-77

19. Thamburaj K, Radhakrishnan VV, Thomas B, et al. Intratumoral microhemorrhages on $\mathrm{T} 2^{\star}$-weighted gradient-echo imaging helps differentiate vestibular schwannoma from meningioma. AJNR Am J Neuroradiol 2008;29:552-57

20. Gasparotti R, Pinelli L, Liserre R. New MR sequences in daily practice: susceptibility weighted imaging: a pictorial essay. Insights Imaging 2011;2:335-47

21. Brisse HJ, Guesmi M, Aerts I, et al. Relevance of CT and MRI in retinoblastoma for the diagnosis of postlaminar invasion with normal-size optic nerve: a retrospective study of 150 patients with histological comparison. Pediatr Radiol 2007;37:649-56 